\title{
Superfast-Trainable Multi-Class Probabilistic Classifier by Least-Squares Posterior Fitting
}

\author{
Masashi SUGIYAMA $^{\dagger, \dagger \dagger a)}$, Senior Member
}

\begin{abstract}
SUMMARY Kernel logistic regression (KLR) is a powerful and flexible classification algorithm, which possesses an ability to provide the confidence of class prediction. However, its training-typically carried out by (quasi-)Newton methods-is rather time-consuming. In this paper, we propose an alternative probabilistic classification algorithm called LeastSquares Probabilistic Classifier (LSPC). KLR models the class-posterior probability by the log-linear combination of kernel functions and its parameters are learned by (regularized) maximum likelihood. In contrast, LSPC employs the linear combination of kernel functions and its parameters are learned by regularized least-squares fitting of the true class-posterior probability. Thanks to this linear regularized least-squares formulation, the solution of LSPC can be computed analytically just by solving a regularized system of linear equations in a class-wise manner. Thus LSPC is computationally very efficient and numerically stable. Through experiments, we show that the computation time of LSPC is faster than that of KLR by two orders of magnitude, with comparable classification accuracy.

key words: probabilistic classification, kernel logistic regression, classposterior probability, squared-loss
\end{abstract}

\section{Introduction}

The support vector machine (SVM) [7], [35] is a popular method for classification. Various computationally efficient algorithms for training SVM with massive datasets have been proposed so far (see [5], [6], [11]-[13], [17], [18], [26], [28], [31]-[34] and many other softwares available online). However, SVM cannot provide the confidence of class prediction since it only learns the decision boundaries between different classes. To cope with this problem, several postprocessing methods have been developed for approximately computing the class-posterior probability [27], [36].

On the other hand, logistic regression (LR) is a classification algorithm that can naturally give the confidence of class prediction since it directly learns the class-posterior probabilities [15]. Recently, various efficient algorithms for training LR models specialized in sparse data have been developed [10], [23].

Applying the kernel trick to LR as done in SVM, one can easily obtain a non-linear classifier with probabilistic outputs, called kernel logistic regression (KLR). Since the kernel matrix is often dense (e.g., Gaussian kernels), the state-of-the-art LR algorithms for sparse data are not

Manuscript received November 18, 2009.

Manuscript revised February 10, 2010.

${ }^{\dagger}$ The author is with Tokyo Institute of Technology, Tokyo, 152-8552 Japan.

${ }^{\dagger \dagger}$ The author is with PRESTO, Japan Science and Technology Agency, Tokyo, 152-8552 Japan.

a)E-mail: sugi@cs.titech.ac.jp

http://sugiyama-www.cs.titech.ac.jp/ sugi/

DOI: $10.1587 /$ transinf.E93.D.2690 applicable to KLR. Thus, in order to train KLR classifiers, standard non-linear optimization techniques such as Newton's method (more specifically, iteratively reweighted least-squares) and quasi-Newton methods (for example, the Broyden-Fletcher-Goldfarb-Shanno (BFGS) method) seem to be commonly used in practice [15], [24]. Although the performance of these general-purpose non-linear optimization techniques has been improved together with the evolution of computer environment in the last decade, computing the KLR solution is still challenging when the number of training samples is large. The purpose of this paper is to propose an alternative probabilistic classification method that can be trained very efficiently.

Our proposed method is called the Least-Squares Probabilistic Classifier (LSPC). In LSPC, we use a linear combination of Gaussian kernels centered at training points as a model of class-posterior probabilities. Then we fit this model to the true class-posterior probability by leastsquares*. An advantage of this linear least-squares formulation is that consistency is guaranteed without taking into account the normalization factor. In contrast, normalization is essential in the maximum-likelihood LR formulation; otherwise the likelihood tends to infinity. Thanks to the simplification brought by excluding the normalization factor from the optimization criterion, we can compute the globally optimal solution of LSPC analytically just by solving a system of linear equations.

Furthermore, we show that the use of a linear combination of kernel functions in LSPC allows us to learn the parameters in a class-wise manner. This highly contributes to further reducing the computational cost particularly in multi-class classification scenarios. Through experiments, we show that LSPC is computationally much more efficient than KLR with comparable accuracy.

\section{Least-Squares Approach to Probabilistic Classifica- tion}

In this section, we formulate the problem of probabilistic classification and give a new method in the least-squares framework.

\footnotetext{
${ }^{*}$ A least-squares formulation has been employed for improving the computational efficiency of SVMs [13], [28], [31]. However, these approaches deal with deterministic classification, not probabilistic classification.
} 


\subsection{Problem Formulation}

Let $\mathcal{X}\left(\subset \mathbb{R}^{d}\right)$ be the input domain, where $d$ is the dimensionality of the input domain. Let $\mathcal{Y}=\{1, \ldots, c\}$ be the set of labels, where $c$ is the number of classes. Let us consider a joint probability distribution on $\mathcal{X} \times \mathcal{Y}$ with joint probability density $p(\boldsymbol{x}, y)$. Suppose that we are given $n$ independent and identically distributed (i.i.d.) paired samples of input $\boldsymbol{x}$ and output $y$ :

$$
\left\{\left(\boldsymbol{x}_{i}, y_{i}\right) \in \mathcal{X} \times \mathcal{Y}\right\}_{i=1}^{n} .
$$

The goal is to estimate the class-posterior probability $p(y \mid \boldsymbol{x})$ from the samples $\left\{\left(\boldsymbol{x}_{i}, y_{i}\right)\right\}_{i=1}^{n}$. The class-posterior probability allows us to classify test sample $x$ to class $\widehat{y}$ with confidence $p(\hat{y} \mid \boldsymbol{x})$ :

$$
\widehat{y}:=\underset{y}{\operatorname{argmax}} p(y \mid \boldsymbol{x}) .
$$

Let us denote the marginal density of $\boldsymbol{x}$ by $p(\boldsymbol{x})$ and we assume that it is strictly positive:

$$
p(\boldsymbol{x})>0 \text { for all } \boldsymbol{x} \in \mathcal{X} .
$$

Then, by definition, the class-posterior probability $p(y \mid \boldsymbol{x})$ can be expressed as

$$
p(y \mid \boldsymbol{x})=\frac{p(\boldsymbol{x}, y)}{p(\boldsymbol{x})} .
$$

This expression will be utilized in the derivation of the proposed method below.

\subsection{Linear Least-Squares Fitting of Class-Posterior Prob- ability}

Here we introduce our least-squares fitting idea. We begin with the formulation for learning the class-posterior probability $p(y \mid \boldsymbol{x})$ as a function of both $\boldsymbol{x}$ and $y$, i.e., the classposterior probabilities for all classes are learned simultaneously. Then in Sect. 2.3, we show that this simultaneous learning problem can be decomposed into independent class-wise learning problems, which highly contributes to reducing the computational cost.

We model the class-posterior probability $p(y \mid x)$ by the following linear model:

$$
q(y \mid \boldsymbol{x} ; \boldsymbol{\alpha}):=\sum_{\ell=1}^{b} \alpha_{\ell} \phi_{\ell}(\boldsymbol{x}, y)=\boldsymbol{\alpha}^{\top} \boldsymbol{\phi}(\boldsymbol{x}, y),
$$

where $^{\top}$ denotes the transpose of a matrix or a vector,

$$
\boldsymbol{\alpha}=\left(\alpha_{1}, \ldots, \alpha_{b}\right)^{\top}
$$

are parameters to be learned from samples, and

$$
\boldsymbol{\phi}(\boldsymbol{x}, y)=\left(\phi_{1}(\boldsymbol{x}, y), \ldots, \phi_{b}(\boldsymbol{x}, y)\right)^{\top}
$$

are basis functions such that

$$
\phi(\boldsymbol{x}, y) \geq \mathbf{0}_{b} \text { for all }(\boldsymbol{x}, y) \in \mathcal{X} \times \mathcal{Y} .
$$

$\mathbf{0}_{b}$ denotes the $b$-dimensional vector with all zeros and the inequality for vectors is applied in an element-wise manner. We explain how the basis functions $\boldsymbol{\phi}(\boldsymbol{x}, \boldsymbol{y})$ are practically chosen in Sect. 2.3.

We determine the parameter $\boldsymbol{\alpha}$ in the model $q(y \mid \boldsymbol{x} ; \boldsymbol{\alpha})$ so that the following squared error $J$ is minimized:

$$
\begin{aligned}
J(\boldsymbol{\alpha}):= & \frac{1}{2} \sum_{y=1}^{c} \int(q(y \mid \boldsymbol{x} ; \boldsymbol{\alpha})-p(y \mid \boldsymbol{x}))^{2} p(\boldsymbol{x}) \mathrm{d} \boldsymbol{x} \\
= & \frac{1}{2} \sum_{y=1}^{c} \int q(y \mid \boldsymbol{x} ; \boldsymbol{\alpha})^{2} p(\boldsymbol{x}) \mathrm{d} \boldsymbol{x} \\
& -\sum_{y=1}^{c} \int q(y \mid \boldsymbol{x} ; \boldsymbol{\alpha}) p(\boldsymbol{x}, y) \mathrm{d} \boldsymbol{x}+\text { Const. } \\
= & \frac{1}{2} \boldsymbol{\alpha}^{\top} \boldsymbol{H} \boldsymbol{\alpha}-\boldsymbol{h}^{\top} \boldsymbol{\alpha}+\text { Const. }
\end{aligned}
$$

where we used Eq. (1). The $b \times b$ matrix $\boldsymbol{H}$ and the $b$ dimensional vector $\boldsymbol{h}$ are defined as

$$
\begin{aligned}
\boldsymbol{H} & :=\sum_{y=1}^{c} \int \boldsymbol{\phi}(\boldsymbol{x}, y) \phi(\boldsymbol{x}, y)^{\top} p(\boldsymbol{x}) \mathrm{d} \boldsymbol{x}, \\
\boldsymbol{h} & :=\sum_{y=1}^{c} \int \boldsymbol{\phi}(\boldsymbol{x}, y) p(\boldsymbol{x}, y) \mathrm{d} \boldsymbol{x} .
\end{aligned}
$$

$\boldsymbol{H}$ and $\boldsymbol{h}$ contain the expectations over unknown densities $p(\boldsymbol{x})$ and $p(\boldsymbol{x}, y)$, so we approximate the expectations by sample averages. Then we have

$$
\begin{aligned}
\widehat{\boldsymbol{H}} & :=\frac{1}{n} \sum_{y=1}^{c} \sum_{i=1}^{n} \boldsymbol{\phi}\left(\boldsymbol{x}_{i}, y\right) \boldsymbol{\phi}\left(\boldsymbol{x}_{i}, y\right)^{\top}, \\
\widehat{\boldsymbol{h}} & :=\frac{1}{n} \sum_{i=1}^{n} \boldsymbol{\phi}\left(\boldsymbol{x}_{i}, y_{i}\right) .
\end{aligned}
$$

Now our optimization criterion is formulated as

$$
\widetilde{\boldsymbol{\alpha}}:=\underset{\boldsymbol{\alpha} \in \mathbb{R}^{b}}{\operatorname{argmin}}\left[\frac{1}{2} \boldsymbol{\alpha}^{\top} \widehat{\boldsymbol{H}} \boldsymbol{\alpha}-\widehat{\boldsymbol{h}}^{\top} \boldsymbol{\alpha}+\lambda \boldsymbol{\alpha}^{\top} \boldsymbol{\alpha}\right],
$$

where a regularizer $\lambda \boldsymbol{\alpha}^{\top} \boldsymbol{\alpha}(\lambda>0)$ is included for regularization purposes. Taking the derivative of the above objective function and equating it to zero, we see that the solution $\widetilde{\boldsymbol{\alpha}}$ can be obtained just by solving the following system of linear equations.

$$
\left(\widehat{\boldsymbol{H}}+\lambda \boldsymbol{I}_{b}\right) \boldsymbol{\alpha}=\widehat{\boldsymbol{h}},
$$

where $\boldsymbol{I}_{b}$ denotes the $b$-dimensional identity matrix. Thus, the solution $\widetilde{\boldsymbol{\alpha}}$ is given analytically as

$$
\widetilde{\boldsymbol{\alpha}}=\left(\widehat{\boldsymbol{H}}+\lambda \boldsymbol{I}_{b}\right)^{-1} \widehat{\boldsymbol{h}} .
$$

Since the class-posterior probability is non-negative by definition, we modify the solution $\widetilde{\boldsymbol{\alpha}}$ as 


$$
\widehat{\boldsymbol{\alpha}}:=\max \left(\mathbf{0}_{b}, \widetilde{\boldsymbol{\alpha}}\right),
$$

where the 'max' operation for vectors is applied in an element-wise manner. We empirically found that if the basis functions and the regularization parameter value are chosen appropriately, e.g., by cross-validation, most elements of $\boldsymbol{\alpha}$ are usually non-negative.

In order to assure that the solution $q(y \mid x ; \widehat{\boldsymbol{\alpha}})$ is a conditional probability, we renormalize the solution in the test phase-given a test input point $\widetilde{\boldsymbol{x}}$, our final solution is given as

$$
\widehat{p}(y \mid \boldsymbol{x}=\widetilde{\boldsymbol{x}})=\frac{\widehat{\boldsymbol{\alpha}}^{\top} \boldsymbol{\phi}(\widetilde{\boldsymbol{x}}, y)}{\sum_{y^{\prime}=1}^{c} \widehat{\boldsymbol{\alpha}}^{\top} \boldsymbol{\phi}\left(\widetilde{\boldsymbol{x}}, y^{\prime}\right)} .
$$

We call the above method Least-Squares Probabilistic Classifier ( $L S P C)$. LSPC can be regarded as an application of a density ratio estimation method called the unconstrained Least-Squares Importance Fitting (uLSIF) [19] to probabilistic classification. Thus all the theoretical properties of uLSIF such as consistency, the rate of convergence, and numerical stability [20], [21] may be directly translated into the current context.

\subsection{Basis Function Design}

A naive choice of basis functions $\phi(x, y)$ would be a kernel model, i.e., for some kernel function $K^{\prime}$,

$$
q(y \mid x ; \boldsymbol{\alpha})=\sum_{y^{\prime}=1}^{c} \sum_{\ell=1}^{n} \alpha_{\ell}^{\left(y^{\prime}\right)} K^{\prime}\left(\boldsymbol{x}, \boldsymbol{x}_{\ell}, y, y^{\prime}\right),
$$

which contains $c n$ parameters. For this model, the computational complexity for solving Eq. (2) is $O\left(c^{3} n^{3}\right)$.

Here we propose to separate input $\boldsymbol{x}$ and output $y$, and use the delta kernel for $y$ (as in KLR):

$$
q(y \mid \boldsymbol{x} ; \boldsymbol{\alpha})=\sum_{y^{\prime}=1}^{c} \sum_{\ell=1}^{n} \alpha_{\ell}^{\left(y^{\prime}\right)} K\left(\boldsymbol{x}, \boldsymbol{x}_{\ell}\right) \delta_{y, y^{\prime}},
$$

where $K$ is a kernel function for $\boldsymbol{x}$ and $\delta_{y, y^{\prime}}$ is the Kronecker delta:

$$
\delta_{y, y^{\prime}}= \begin{cases}1 & \text { if } y=y^{\prime} \\ 0 & \text { otherwise }\end{cases}
$$

This model choice actually allows us to speed up the computation of LSPC significantly since all the calculations can be carried out separately in a class-wise manner. Indeed, the above model for class $y$ is expressed as

$$
q(y \mid x ; \boldsymbol{\alpha})=\sum_{\ell=1}^{n} \alpha_{\ell}^{(y)} K\left(\boldsymbol{x}, \boldsymbol{x}_{\ell}\right) .
$$

Then the matrix $\widehat{\boldsymbol{H}}$ becomes block-diagonal, as illustrated in Fig. 1 (a). Thus we only need to train a model with $n$ parameters separately $c$ times for each class $y$. In this case, the computational complexity for computing the solution is

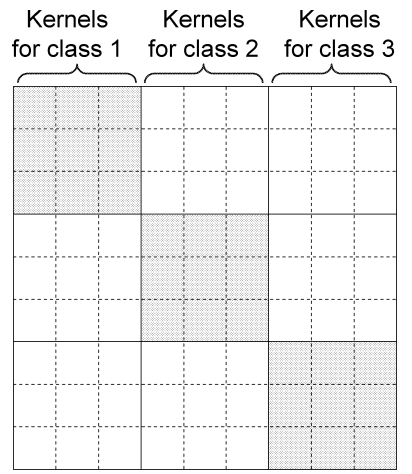

(a) Model (5)

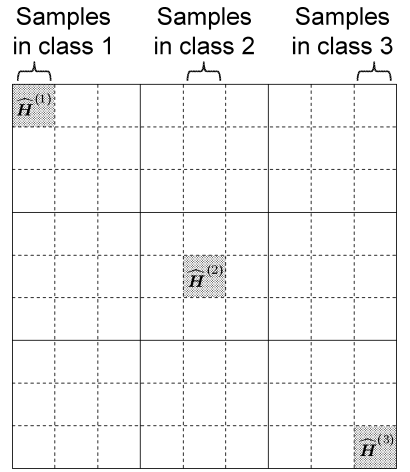

(b) Model (7)
Fig. 1 Structure of matrix $\widehat{\boldsymbol{H}}$ for model (5) and model (7). The number of classes is $c=3$. Suppose training samples $\left\{\left(\boldsymbol{x}_{i}, y_{i}\right)\right\}_{i=1}^{n}$ are sorted according to label $y$. Colored blocks are non-zero and others are zeros. For model (5) consisting of $c$ sets of $n$ basis functions, the matrix $\widehat{\boldsymbol{H}}$ becomes blockdiagonal and thus training can be carried out separately for each block. For model (7) consisting of $c$ sets of $n_{y}$ basis functions, the size of the target block is further reduced.

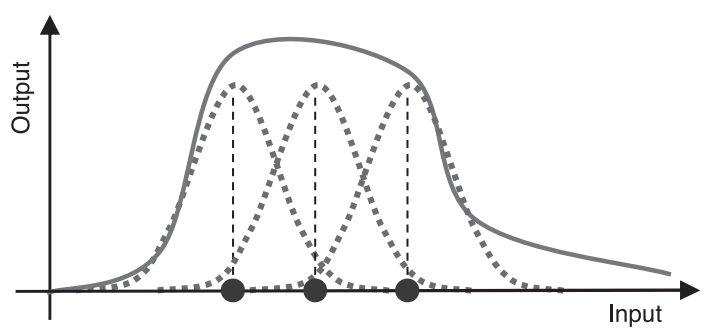

Fig. 2 Heuristic of reducing the number of basis functions-locate Gaussian kernels only at the samples of the target class.

reduced to $O\left(c n^{3}\right)$, which is $c^{2}$ times smaller than the case with general kernel model (4). Thus this approach would be computationally efficient when the number of classes $c$ is large.

Here, we further propose to reduce the number of kernels in model (5). To this end, we focus on a kernel function $K\left(\boldsymbol{x}, \boldsymbol{x}^{\prime}\right)$ that is non-negative for all $\boldsymbol{x}, \boldsymbol{x}^{\prime}(\in \mathcal{X})$ and "localized". Examples of such kernels includes the popular Gaussian kernel [30]:

$$
K\left(\boldsymbol{x}, \boldsymbol{x}^{\prime}\right)=\exp \left(-\frac{\left\|\boldsymbol{x}-\boldsymbol{x}^{\prime}\right\|^{2}}{2 \sigma^{2}}\right) .
$$

Here we propose to reduce the number of kernels by locating the kernels only at samples belonging to the target class:

$$
q(y \mid x ; \boldsymbol{\alpha})=\sum_{\ell=1}^{n_{y}} \alpha_{\ell}^{(y)} K\left(\boldsymbol{x}, \boldsymbol{x}_{\ell}\right),
$$

where $n_{y}$ is the number of training samples in class $y$, and $\left\{\boldsymbol{x}_{i}^{(y)}\right\}_{i=1}^{n_{y}}$ is the training input samples in class $y$.

The rationale behind this model simplification is as follows (Fig. 2). By definition, the class-posterior probability $p(y \mid x)$ takes large values in the regions where samples in class $y$ are dense; conversely, $p(y \mid x)$ takes smaller values (i.e., close to zero) in the regions where samples in class $y$ 


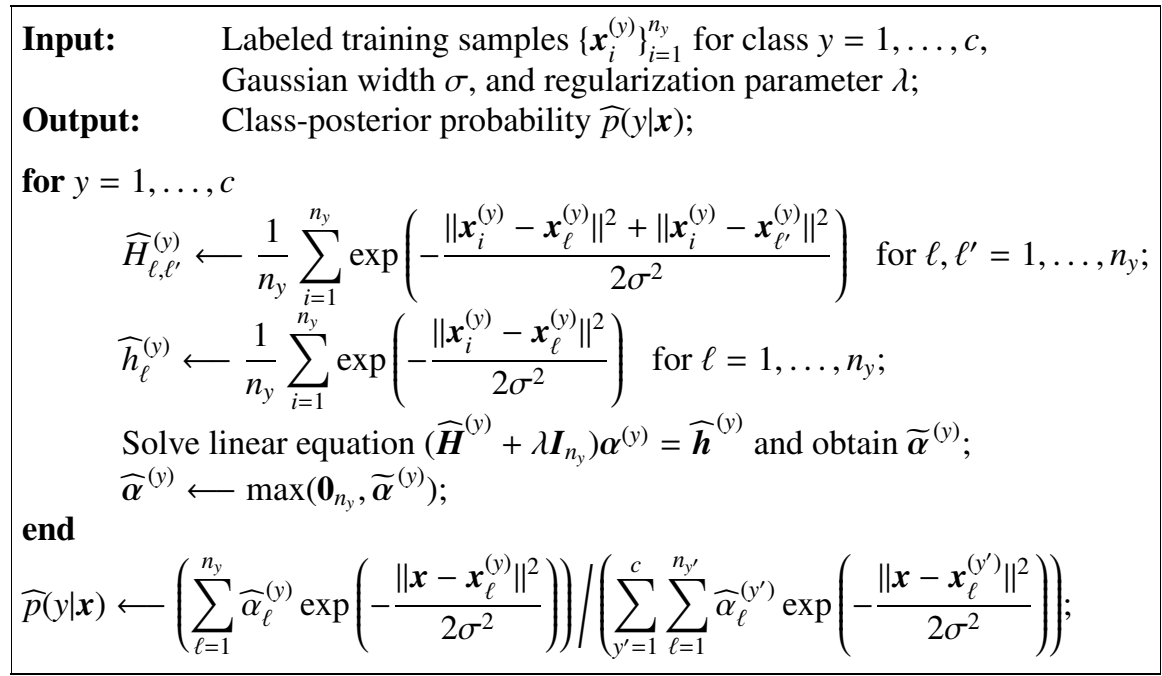

Fig.3 Pseudo code of LSPC for simplified model (7) with Gaussian kernel (6). $\mathbf{0}_{n_{y}}$ denotes the $n_{y}$-dimensional vector with all zeros. The 'max' operation for vectors is applied in an element-wise manner.

are sparse. When a non-negative function is approximated by a Gaussian kernel model, many kernels may be needed in the region where the output of the target function is large; on the other hand, only a small number of kernels would be enough in the region where the output of the target function is close to zero. Following this heuristic, many kernels are allocated in the region where $p(y \mid x)$ takes large values, which can be achieved by Eq. (7).

This model simplification allows us to further reduce the computational cost since the size of the target blocks in matrix $\widehat{\boldsymbol{H}}$ is further reduced, as illustrated in Fig. 1 (b). In order to learn the $n_{y}$-dimensional parameter vector

$$
\boldsymbol{\alpha}^{(y)}=\left(\alpha_{1}^{(y)}, \ldots, \alpha_{n_{y}}^{(y)}\right)^{\top}
$$

for each class $y$, we only need to solve the following system of $n_{y}$ linear equations:

$$
\left(\widehat{\boldsymbol{H}}^{(y)}+\lambda \boldsymbol{I}_{n_{y}}\right) \boldsymbol{\alpha}^{(y)}=\widehat{\boldsymbol{h}}^{(y)},
$$

where $\widehat{\boldsymbol{H}}^{(y)}$ is the $n_{y} \times n_{y}$ matrix, and $\widehat{\boldsymbol{h}}^{(y)}$ is the $n_{y^{-}}$ dimensional vector defined as

$$
\begin{aligned}
\widehat{H}_{\ell, \ell^{\prime}}^{(y)} & :=\frac{1}{n_{y}} \sum_{i=1}^{n_{y}} K\left(\boldsymbol{x}_{i}^{(y)}, \boldsymbol{x}_{\ell}^{(y)}\right) K\left(\boldsymbol{x}_{i}^{(y)}, \boldsymbol{x}_{\ell^{\prime}}^{(y)}\right), \\
\widehat{h}_{\ell}^{(y)} & :=\frac{1}{n_{y}} \sum_{i=1}^{n_{y}} K\left(\boldsymbol{x}_{i}^{(y)}, \boldsymbol{x}_{\ell}^{(y)}\right) .
\end{aligned}
$$

Let $\widetilde{\boldsymbol{\alpha}}^{(y)}$ be the solution of Eq. (8). Then our final solution is given by

$$
\widehat{p}(y \mid \boldsymbol{x})=\frac{\sum_{\ell=1}^{n_{y}} \widehat{\alpha}_{\ell}^{(y)} K\left(\boldsymbol{x}, \boldsymbol{x}_{\ell}^{(y)}\right)}{\sum_{y^{\prime}=1}^{c} \sum_{\ell=1}^{n_{y^{\prime}}} \widehat{\alpha}_{\ell}^{\left(y^{\prime}\right)} K\left(\boldsymbol{x}, \boldsymbol{x}_{\ell}^{\left(y^{\prime}\right)}\right)},
$$

where

$$
\widehat{\boldsymbol{\alpha}}^{(y)}:=\max \left(\mathbf{0}_{n_{y}}, \widetilde{\boldsymbol{\alpha}}^{(y)}\right) .
$$

For the simplified model (7), the computational complexity for computing the solution is $O\left(c n_{y}^{3}\right)$. When $n_{y}=n / c$ for all $y$, this is equal to $O\left(c^{-2} n^{3}\right)$, which is $c^{5}$ times smaller than the case with general kernel model (4) and is $c^{3}$ times smaller than the case with delta-kernel model (5). Thus this approach is computationally highly efficient for multi-class problems.

A pseudo code of the simplest LSPC implementation for Gaussian kernels is summarized in Fig. 3. Its MATLAB $^{\circledR}$ implementation is available from http://sugiyama-www.cs.titech.ac.jp/rsugi/software/LSPC/

\section{Experiments}

In this section, we experimentally compare the performance of the following classification methods:

- LSPC: LSPC with model (7).

- LSPC(full): LSPC with model (5).

- KLR: $\ell_{2}$-penalized kernel logistic regression with Gaussian kernels. We used a MATLAB ${ }^{\circledR}$ implementation included in the 'minFunc' package [29], which uses limited-memory BFGS updates with Shanno-Phua scaling in computing the step direction and a bracketing line-search for a point satisfying the strong Wolfe conditions to compute the step direction.

- PSVM: A probabilistic version of the support vector machine with Gaussian kernels based on pairwise coupling [36]. We used the LIBSVM implementation [5].

- SVM: A support vector machine with Gaussian kernels [7], [35]. We used the LIBSVM implementation [5], which is a sequential-minimal optimization algorithm [11], [26] using the 'one-versus-one' strategy for multi-class classification [16]. Note that SVM cannot output class-posterior probabilities-we included it 

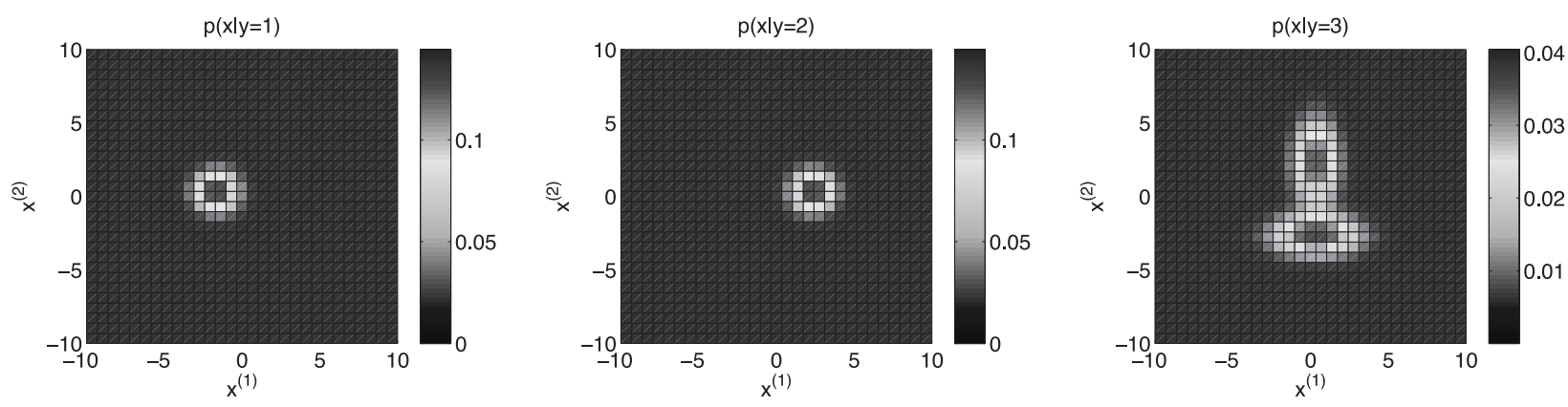

Fig. 4 Illustrative examples. Class-conditional sample densities $p(\boldsymbol{x} \mid y)$.

in our comparison just for a reference.

When we fed data to learning algorithms, the input samples were normalized in an element-wise manner so that each element has mean zero and unit variance. The Gaussian width $\sigma$ and the regularization parameter $\lambda$ for all the methods are chosen based on 2-fold cross-validation from

$$
\begin{aligned}
& \sigma \in\left\{\frac{1}{10} m, \frac{1}{5} m, \frac{1}{2} m, \frac{2}{3} m, m, \frac{3}{2} m, 2 m, 5 m, 10 m\right\}, \\
& \lambda \in\left\{10^{-2}, 10^{-1.5}, 10^{-1}, 10^{-0.5}, 10^{0}\right\},
\end{aligned}
$$

where

$$
m:=\operatorname{median}\left(\left\{\left\|\boldsymbol{x}_{i}-\boldsymbol{x}_{j}\right\|\right\}_{i, j=1}^{n}\right) .
$$

For PSVM and SVM, we set $C=1 / \lambda$.

\subsection{Illustrative Examples}

First, we illustrate the behavior of each method using a toy dataset.

We set the dimension of the input space to $d=2$ and the number of classes to $c=3$. We independently drew samples in each class from the following class-conditional sample densities (see Fig. 4):

$$
\begin{aligned}
p(x \mid y=1)= & N\left(x ;\left[\begin{array}{c}
-2 \\
0
\end{array}\right],\left[\begin{array}{ll}
1 & 0 \\
0 & 1
\end{array}\right]\right), \\
p(x \mid y=2)= & N\left(x ;\left[\begin{array}{l}
2 \\
0
\end{array}\right],\left[\begin{array}{ll}
1 & 0 \\
0 & 1
\end{array}\right]\right), \\
p(x \mid y=3)= & \frac{1}{2} N\left(x ;\left[\begin{array}{c}
0 \\
-3
\end{array}\right],\left[\begin{array}{ll}
4 & 0 \\
0 & 1
\end{array}\right]\right) \\
& +\frac{1}{2} N\left(x ;\left[\begin{array}{l}
0 \\
2
\end{array}\right],\left[\begin{array}{ll}
1 & 0 \\
0 & 4
\end{array}\right]\right),
\end{aligned}
$$

where $N(\boldsymbol{x} ; \boldsymbol{\mu}, \boldsymbol{\Sigma})$ denotes the Gaussian density with mean $\boldsymbol{\mu}$ and covariance matrix $\boldsymbol{\Sigma}$. We set the class-prior probabilities $p(y)$ as

$$
p(y)= \begin{cases}1 / 4 & \text { if } y=1,2, \\ 1 / 2 & \text { if } y=3,\end{cases}
$$

and we set the number of training samples to $n=200$. Generated samples are plotted in Fig. 5.

The true class-posterior probabilities $p(y \mid x) \quad(\propto$ $p(\boldsymbol{x} \mid y) p(y))$, their estimates obtained by LSPC, LSPC(full), KLR, and PSVM are depicted in Fig. 6. The plots show that all the methods approximate the true class-posterior probabilities well in the training region (say, $[-5,5]^{2}$ ). However, the output outside the training region is substantially different in LSPC and KLR. This is induced by the difference of the models - a linear combination of Gaussian kernels is used in LSPC while its exponent is used in KLR. Outside the training region, there is no kernel and thus a linear combination of Gaussian kernels takes values close to zero (note that the values are not exactly zero since Gaussian tails extended from training regions remain everywhere); then typically one of the classes takes a value close to one and the others tend to zero outside the training regions. On the other hand, KLR outputs values close to one outside the training region since $\exp (0)=1$; then they are normalized and thus are reduced to $1 / c$.

The classification results based on the true classposterior probabilities and their estimates obtained by LSPC, LSPC(full), KLR, PSVM, and SVM are plotted in Fig. 5. This shows that all the method gave reasonable classification results.

\subsection{Performance Comparison}

Next, we evaluate the classification accuracy and computation time of each method using the following multi-class classification datasets taken from the LIBSVM web page [5]:

- mnist: Input dimensionality is 717 and the number of classes is 10 .

- usps: Input dimensionality is 256 and the number of classes is 10 .

- satimage: Input dimensionality is 36 and the number of classes is 6 .

- letter: Input dimensionality is 16 and the number of classes is 26.

We investigated the classification accuracy and computation time of LSPC, LSPC(full), KLR, PSVM, and SVM. For given $n$ and $c$, we randomly chose $n_{y}=\lfloor n / c\rfloor$ training samples from each class $y$, where $\lfloor t\rfloor$ is the largest integer not greater than $t$. In the first set of experiments, we fixed the number of classes $c$ to the original number shown above and changed the number of training samples 

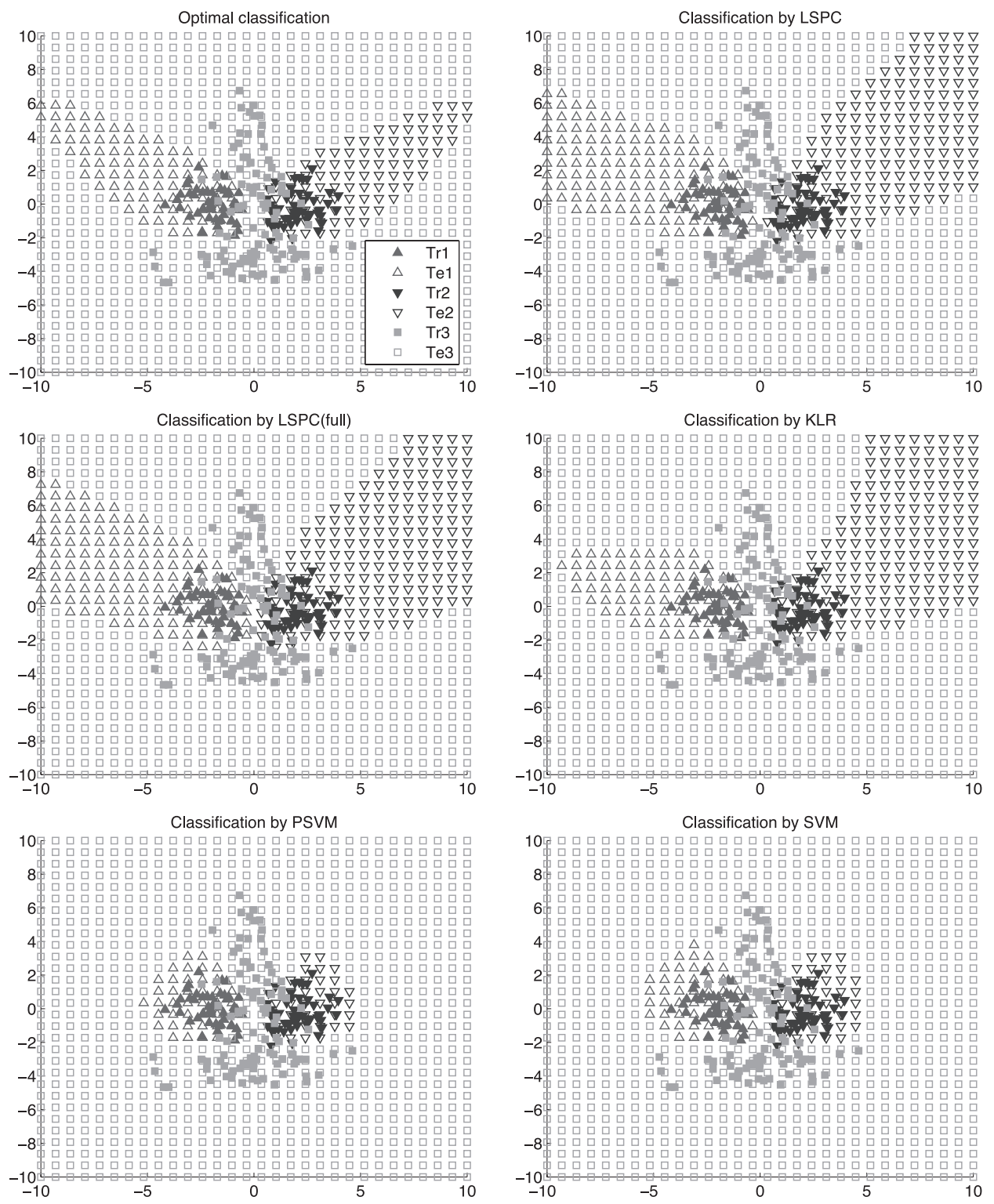

Fig. 5 Illustrative examples. Training samples are plotted with filled symbols. Unfilled symbols denote the classification results based on the true class-posterior probabilities and their estimates obtained by LSPC, LSPC(full), KLR, PSVM, and SVM.

as $n=100,200,500,1000,2000$. In the second set of experiments, we fixed the number of training samples to $n=1000$ and changed the number of classes $c$-samples only in the first $c$ classes in the dataset are used. The classification accuracy is evaluated using 100 test samples randomly chosen from each class. The computation time is measured by the CPU computation time required for training each classifier when the Gaussian width and the regularization parameter chosen by cross-validation were used.

The experimental results are summarized in Fig. 7 and Fig. 8. The left column in Fig. 7 shows that when $n$ is increased, the classification error for all the methods tends to decrease. LSPC, LSPC(full), and KLR performed similarly well, but PSVM and SVM did not work properly. The right column in Fig. 7 shows that when $n$ is increased, the com- putation time tends to grow for all the methods. LSPC is faster than that of KLR by two orders of magnitude, and that of LSPC(full) and PSVM by an order of magnitude in most cases. The left column in Fig. 8 shows that when $c$ is increased, the classification error tends to increase for all the methods. LSPC, LSPC(full), and KLR behaved similarly well, while PSVM and SVM performed poorly. The right column in Fig. 8 shows that when $c$ is increased, the computation time of LSPC(full), KLR, PSVM, and SVM tend to slightly grow, while that of LSPC tends to decrease. This happens because the number of samples in each class decreases when $c$ is increased, and the computation time of LSPC is governed by the number of samples in each class, not by the total number of samples (see Sect. 2.3).

Overall, the computation of LSPC was shown to be 

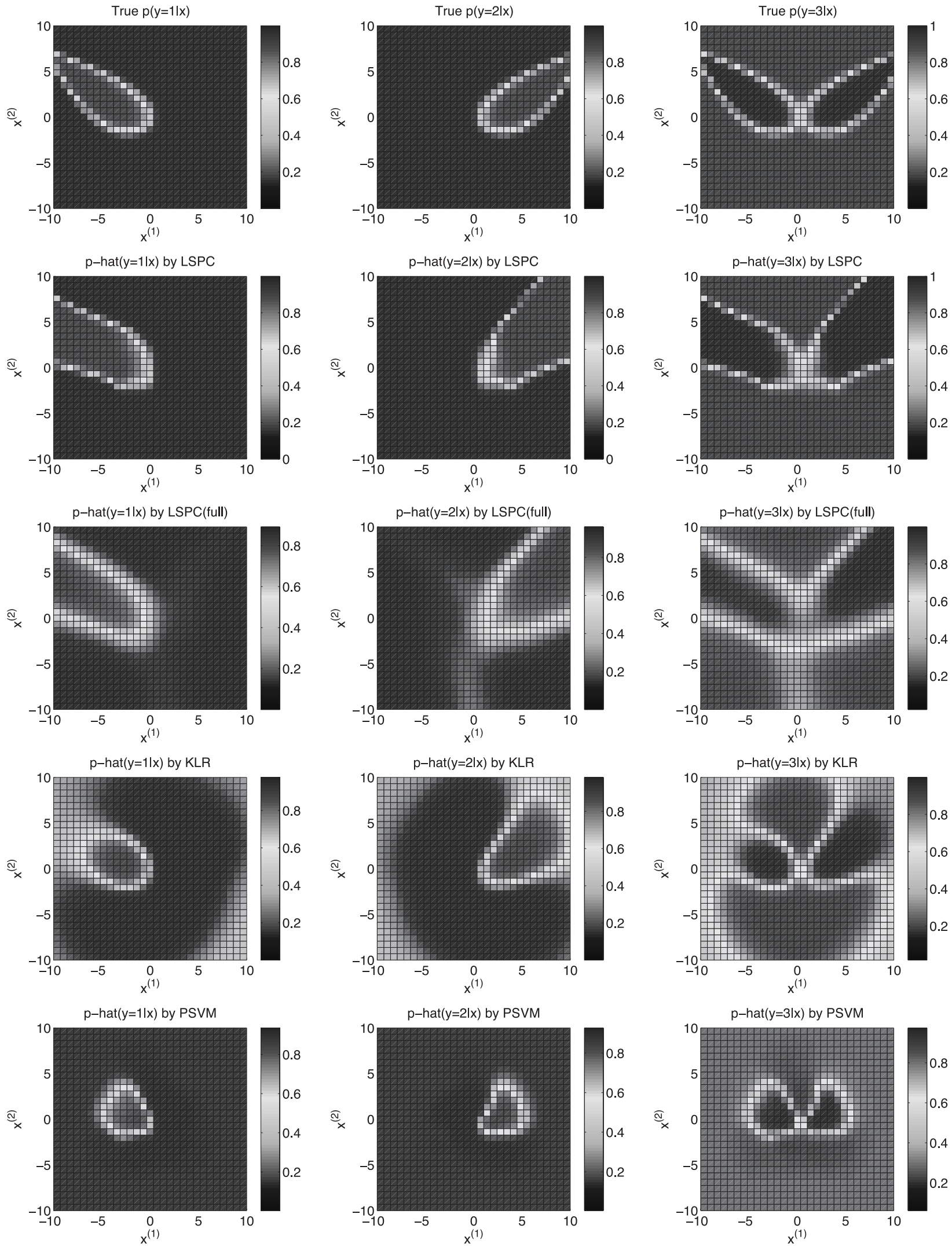

Fig. 6 Illustrative examples. The plots show the true class-posterior probabilities $p(y \mid x)$, their estimates by LSPC, LSPC(full), KLR, and PSVM from top to bottom, and $y=1,2,3$ from left to right. 

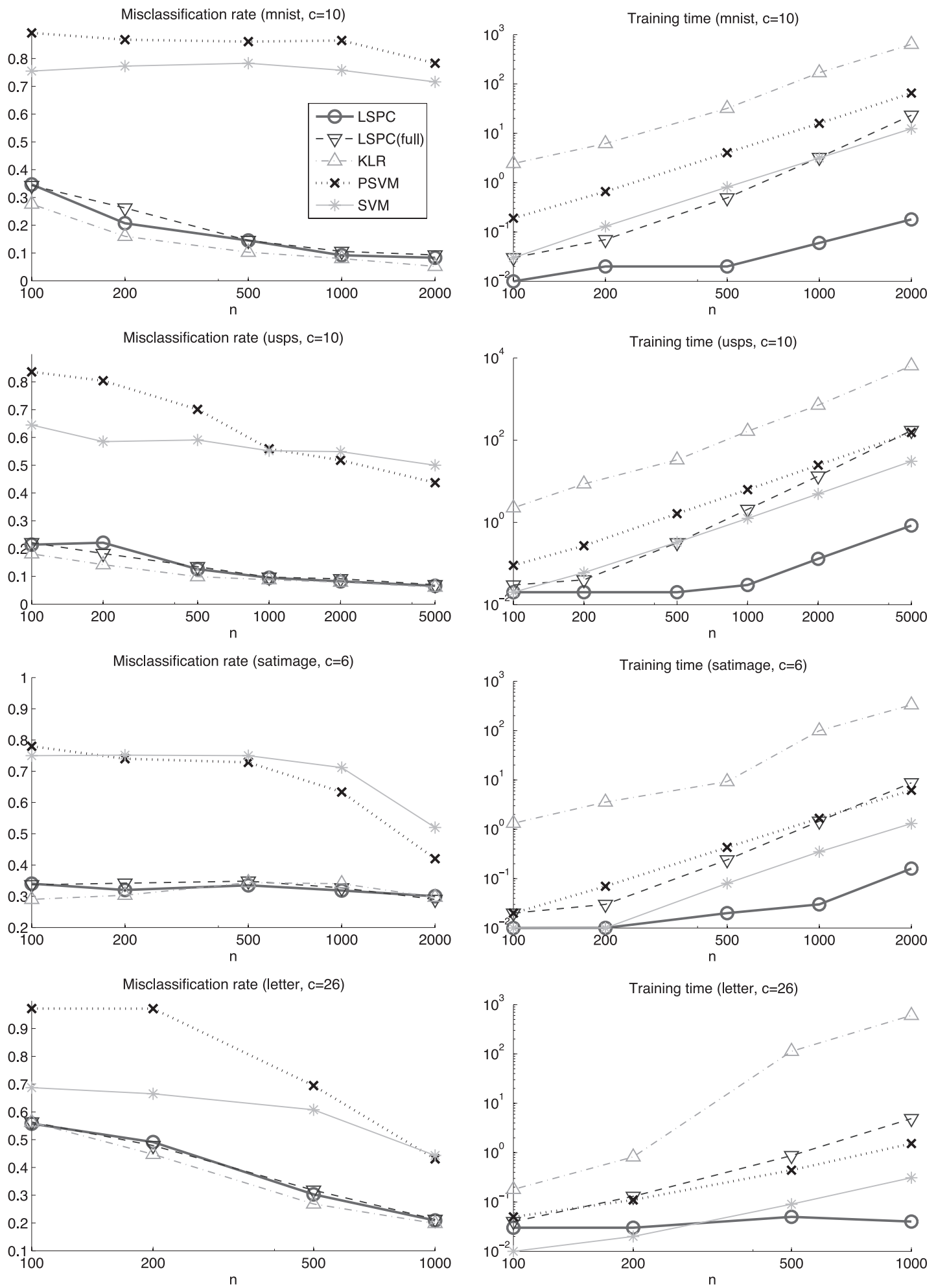

Fig. 7 Misclassification rate (in percent, left) and computation time (in second, right) as functions of the number of training samples $n$. From top to bottom, the graphs correspond to the 'mnist', 'usps', 'satimage', and 'letter' datasets. The graphs of computation time of LSPC for small $n$ are flat (e.g., the 'usps' and 'satimage' datasets) since the computation time was almost zero and was rounded up to the smallest unit $10^{-2}$ in the CPU time counter. 

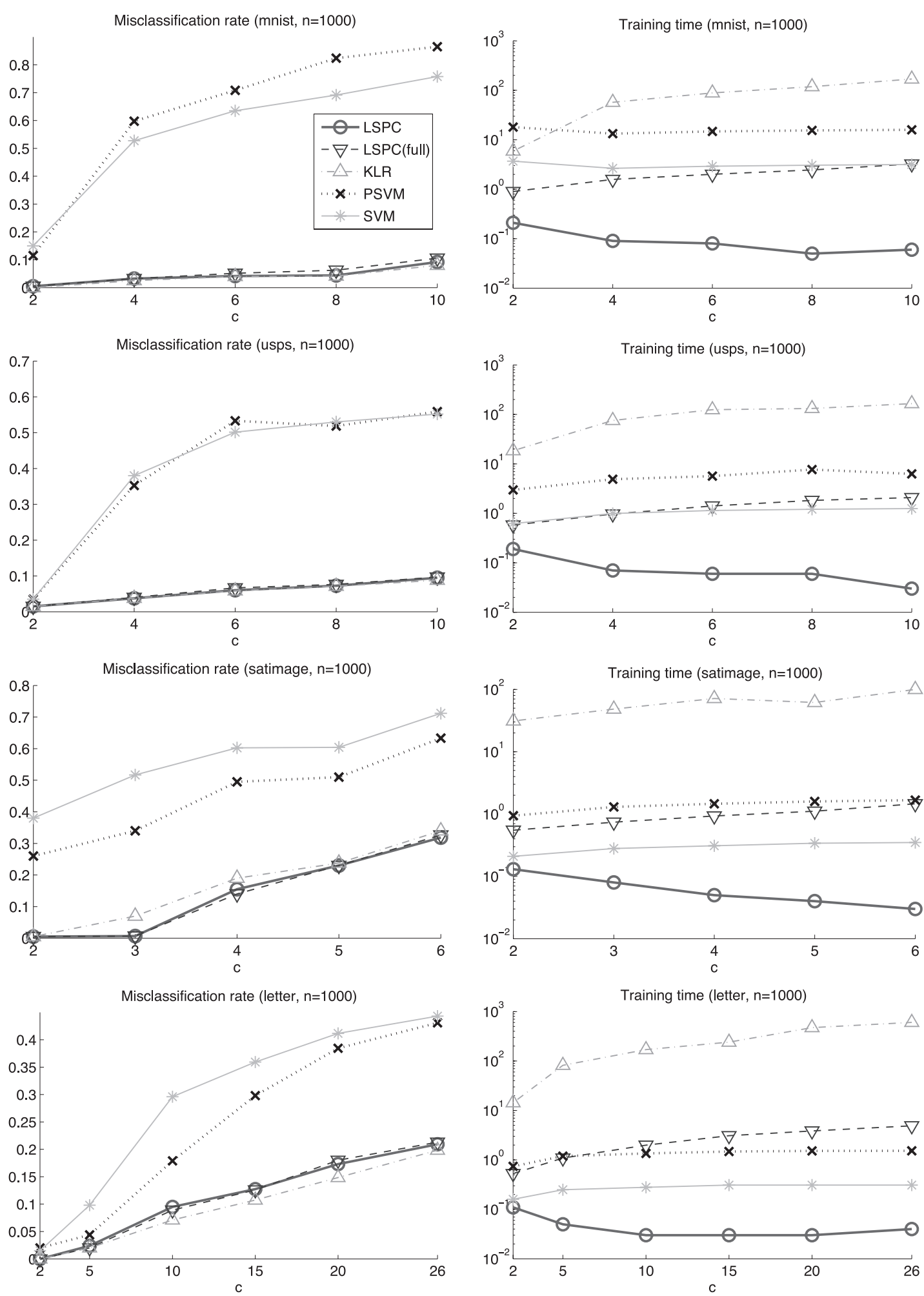

Fig. 8 Misclassification rate (in percent, left) and computation time (in second, right) as functions of the number of classes $c$. From top to bottom, the graphs correspond to the 'mnist', 'usps', 'satimage', and 'letter' datasets. 
faster than that of KLR by two orders of magnitude, while LSPC and KLR were shown to be comparable to each other in classification accuracy. LSPC and LSPC(full) were shown to possess similar classification performance, and thus a computationally efficient version, LSPC, would be more preferable in practice. We also found that PSVM and SVM perform poorly in multi-class scenarios. Thus the use of the proposed LSPC would be promising particularly in multi-class scenarios.

\section{Discussion and Conclusion}

Recently, various efficient algorithms for computing the solution of logistic regression have been developed for highdimensional sparse data [10], [23]. However, for dense data, using standard non-linear optimization techniques such as Newton's method or quasi-Newton methods seem to be a common choice [15], [24]. The performance of these general-purpose non-linear optimizers has been improved in the last decade, but computing the solution of logistic regression for a large number of dense training samples is still a challenge problem.

In this paper, we proposed a simple probabilistic classification algorithm called Least-Squares Probabilistic Classifier (LSPC). LSPC employs a linear combination of Gaussian kernels centered at training points for modeling the class-posterior probability and the parameters are learned by least-squares. Notable advantages of LSPC are that its solution can be computed analytically just by solving a system of linear equations and training can be carried out separately in a class-wise manner. In experiments, we showed that LSPC is faster than kernel logistic regression (KLR) in computation time by two orders of magnitude, with comparable accuracy.

The computational efficiency of LSPC was brought by the combination of appropriate model choice and loss function. More specifically, KLR uses a log-linear combination of kernel functions and its parameters are learned by regularized maximum likelihood. In this log-linear maximum likelihood formulation, normalization of the model is essential to avoid the likelihood diverging to infinity. Thus the likelihood function tends to be complicated and numerically solving the optimization problem may be unavoidable. On the other hand, in LSPC, we chose a linear combination of Gaussian kernel functions for modeling the class-posterior probability and its parameters are learned by regularized least-squares. This combination allowed us to obtain the solution analytically. When Newton's method (more specifically, iteratively reweighted least-squares) is used for learning the KLR model, a system of linear equations needs to be solved in every iteration until convergence [15]. On the other hand, LSPC requires to solve a system of linear equations only once.

We chose to separate the kernel for inputs and outputs, and adopted the delta kernel for outputs (see Eq. (5)). This allowed us to perform the training of LSPC in a class-wise manner. We showed that this contributes to reducing the training time particularly in multi-class classification problems. We note that this model choice is essentially the same as that of $\mathrm{KLR}^{\dagger}$.

We further proposed to reduce the number of kernels when "localized" kernels such as the Gaussian kernel (6) is used. Through the experimental evaluation in Sect. 3, we found that this heuristic model simplification does not degrade the classification accuracy, but reduces the computation time significantly.

It is straightforward to show that solutions for all regularization parameter values (i.e., the regularization path, see [9], [14]) can be computed efficiently in LSPC. Let us consider the eigendecomposition of the matrix $\widehat{\boldsymbol{H}}^{(y)}$ (see Eq. (9)):

$$
\widehat{\boldsymbol{H}}^{(y)}=\sum_{\ell=1}^{n_{y}} \gamma_{\ell} \boldsymbol{\psi}_{\ell} \boldsymbol{\psi}_{\ell}^{\top},
$$

where $\left\{\boldsymbol{\psi}_{\ell}\right\}_{\ell=1}^{n_{y}}$ are the eigenvectors of $\widehat{\boldsymbol{H}}^{(y)}$ associated with the eigenvalues $\left\{\gamma_{\ell}\right\}_{\ell=1}^{n_{y}}$. Then, the solution $\widetilde{\boldsymbol{\alpha}}^{(y)}$ can be expressed as

$$
\widetilde{\boldsymbol{\alpha}}^{(y)}=\left(\widehat{\boldsymbol{H}}^{(y)}+\lambda \boldsymbol{I}_{n_{y}}\right)^{-1} \widehat{\boldsymbol{h}}^{(y)}=\sum_{\ell=1}^{n_{y}} \frac{\widehat{\boldsymbol{h}}^{\top} \boldsymbol{\psi}_{\ell}}{\gamma_{\ell}+\lambda} \boldsymbol{\psi}_{\ell} .
$$

Since $\left(\widehat{\boldsymbol{h}}^{\top} \boldsymbol{\psi}_{\ell}\right) \boldsymbol{\psi}_{\ell}$ is common to all $\lambda$, we can compute the solution $\widetilde{\boldsymbol{\alpha}}^{(y)}$ for all $\lambda$ efficiently by eigendecomposing the matrix $\widehat{\boldsymbol{H}}^{(y)}$ once in advance. Although eigendecomposition of $\widehat{\boldsymbol{H}}^{(y)}$ may be computationally slightly more demanding than solving a system of linear equations of the same size, this approach would be useful, e.g., when computing the solutions for various values of $\lambda$ in the cross-validation procedure.

When $n_{y}$ is large, we may further reduce the computational cost and memory space by using only a subset of kernels.

$$
\begin{aligned}
& q(y \mid \boldsymbol{x} ; \boldsymbol{\alpha})=\sum_{\ell=1}^{b_{y}} \alpha_{\ell}^{(y)} K\left(\boldsymbol{x}, \boldsymbol{c}_{\ell}^{(y)}\right), \\
& \widehat{H}_{\ell, \ell^{\prime}}^{(y)}=\frac{1}{n_{y}} \sum_{i=1}^{n_{y}} K\left(\boldsymbol{x}_{i}^{(y)}, \boldsymbol{c}_{\ell}^{(y)}\right) K\left(\boldsymbol{x}_{i}^{(y)}, \boldsymbol{c}_{\ell^{\prime}}^{(y)}\right), \\
& \widehat{h}_{\ell}^{(y)}=\frac{1}{n_{y}} \sum_{i=1}^{n_{y}} K\left(\boldsymbol{x}_{i}^{(y)}, \boldsymbol{c}_{\ell}^{(y)}\right),
\end{aligned}
$$

where $b_{y}$ is a constant chosen to be smaller than $n_{y}$ and $\left\{\boldsymbol{c}_{\ell}^{(y)}\right\}_{\ell=1}^{b_{y}}$ is a subset of $\left\{\boldsymbol{x}_{\ell}^{(y)}\right\}_{\ell=1}^{n_{y}}$. This would be a useful heuristic when a huge number of samples are available.

Another option for reducing the computation time when the number of samples is very large would be the stochastic gradient descent method [1]. That is, starting from some initial parameter value, gradient descent is car-

${ }^{\dagger}$ The number of LSPC for model (5) is $c n$, while the number of parameters in KLR is $(c-1) n$ since the normalization (sum-to-one) constraint is incorporated in the training phase. 
ried out only for a randomly chosen single sample in each iteration. Since our optimization problem is convex, convergence to the global solution is guaranteed (in the probabilistic sense) by stochastic gradient descent.

The LSPC algorithm was originally designed as a discriminative classifier [25] since it directly learns the classposterior probability $p(y \mid \boldsymbol{x})$. However, through the simplification of models explored in Sect. 2.3, the final solution (10) can be actually interpreted as a generative classifier. That is, via the Bayes law,

$$
p(y \mid \boldsymbol{x}) \propto p(\boldsymbol{x} \mid y) p(y),
$$

the solution (10) is equivalent to approximating $p(y)$ by $n_{y} / n$ and $p(\boldsymbol{x} \mid y)$ by least-squares kernel density estimation for each class $y$. This correspondence would explain why our heuristic model simplification (see Fig. 2) worked well in the experiments in Sect. 3 .

Similar correspondence between discriminative and generative classification can also be observed in linear regression and maximum-likelihood estimation of class-wise Gaussian densities [3], [8]. Thus, further analyzing the LSPC approach as a generative classifier would be an interesting future work for more deeply understanding the mechanism of the LSPC method.

As shown in Eq. (4), instead of the delta kernel, one may use other kernels for class labels. We expect that designing appropriate kernel functions for class labels would be useful for improving the classification performance in the context of multi-task learning [2], [4], [22]. We will pursue this direction in our future work.

\section{Acknowledgments}

The author thanks Dr. Ryota Tomioka for his valuable comments. This work was supported by AOARD, SCAT, and the JST PRESTO program.

\section{References}

[1] S. Amari, "Theory of adaptive pattern classifiers," IEEE Trans. Electronic Computers, vol.EC-16, no.3, pp.299-307, 1967.

[2] B. Bakker and T. Heskes, "Task clustering and gating for Bayesian multitask learning," J. Machine Learning Research, vol.4, pp.83-99, 2003.

[3] C.M. Bishop, Pattern Recognition and Machine Learning, Springer, New York, NY, USA, 2006.

[4] R. Caruana, L. Pratt, and S. Thrun, "Multitask learning," Mach. Learn., vol.28, p.41, 1997.

[5] C.C. Chang and C.J. Lin, "LIBSVM: A library for support vector machines," Tech. Rep., Department of Computer Science, National Taiwan University, 2001. http://www.csie.ntu.edu.tw/ cjlin/libsvm/

[6] R. Collobert and S. Bengio, "SVMTorch: Support vector machines for large-scale regression problems," J. Machine Learning Research, vol.1, pp.143-160, 2001.

[7] C. Cortes and V. Vapnik, "Support vector networks," Mach. Learn., vol.20, pp.273-297, 1995.

[8] R.O. Duda, P.E. Hart, and D.G. Stor, Pattern Classification, Wiley, New York, 2001.

[9] B. Efron, T. Hastie, R. Tibshirani, and I. Johnstone, "Least angle regression," Annals of Statistics, vol.32, no.2, pp.407-499, 2004.
[10] R.E. Fan, K.W. Chang, C.J. Hsieh, X.R. Wang, and C.J. Lin, "LIBLINEAR: A library for large linear classification," J. Machine Learning Research, vol.9, pp.1871-1874, 2008.

[11] R.E. Fan, P.H. Chen, and C.J. Lin, "Working set selection using second order information for training SVM," J. Machine Learning Research, vol.6, pp.1889-1918, 2005.

[12] V. Franc and S. Sonnenburg, "Optimized cutting plane algorithm for large-scale risk minimization," J. Machine Learning Research, vol.10, pp.2157-2192, 2009.

[13] G.M. Fung and O.L. Mangasarian, "Multicategory proximal support vector machine classifiers," Mach. Learn., vol.59, no.1-2, pp.77-97, 2005.

[14] T. Hastie, S. Rosset, R. Tibshirani, and J. Zhu, "The entire regularization path for the support vector machine," J. Machine Learning Research, vol.5, pp.1391-1415, 2004.

[15] T. Hastie, R. Tibshirani, and J. Friedman, The Elements of Statistical Learning: Data Mining, Inference, and Prediction, Springer, New York, 2001.

[16] C.W. Hsu and C.J. Lin, "A comparison of methods for multi-class support vector machines," IEEE Trans. Neural Netw., vol.13, no.2, pp.415-425, 2002.

[17] T. Joachims, "Making large-scale SVM learning practical," in Advances in Kernel Methods-Support Vector Learning, ed. B. Schölkopf, C.J.C. Burges, and A.J. Smola, pp.169-184, The MIT Press, Cambridge, MA, 1999.

[18] T. Joachims, "Training linear SVMs in linear time," ACM SIGKDD International Conference on Knowledge Discovery and Data Mining (KDD2006), pp.217-226, 2006.

[19] T. Kanamori, S. Hido, and M. Sugiyama, "A least-squares approach to direct importance estimation," J. Machine Learning Research, vol.10, pp.1391-1445, July 2009.

[20] T. Kanamori, T. Suzuki, and M. Sugiyama, "Condition number analysis of kernel-based density ratio estimation," Tech. Rep., arXiv, 2009.

[21] T. Kanamori, T. Suzuki, and M. Sugiyama, "Theoretical analysis of density ratio estimation," IEICE Trans. Fundamentals, vol.E93-A, no.4, pp.787-798, April 2010.

[22] T. Kato, H. Kashima, M. Sugiyama, and K. Asai, "Conic programming for multi-task learning," IEEE Trans. Knowl. Data Eng., vol.22, no.7, pp.957-968, 2010.

[23] K. Koh, S.J. Kim, and S.P. Boyd, "An interior-point method for large-scale $l_{1}$-regularized logistic regression," J. Machine Learning Research, vol.8, pp.1519-1555, 2007.

[24] T.P. Minka, "A comparison of numerical optimizers for logistic regression,” Tech. Rep., Microsoft Research, 2007.

[25] A.Y. Ng and M.I. Jordan, "On discriminative vs. generative classifiers: A comparison of logistic regression and naive bayes," Advances in Neural Information Processing Systems 14, ed. T.G. Dietterich, S. Becker, and Z. Ghahramani, MIT Press, Cambridge, MA, 2002.

[26] J. Platt, "Fast training of support vector machines using sequential minimal optimization," in Advances in Kernel Methods-Support Vector Learning, ed. B. Schölkopf, C.J.C. Burges, and A.J. Smola, pp.169-184, The MIT Press, Cambridge, MA, 1999.

[27] J. Platt, "Probabilities for SV machines," in Advances in Large Margin Classifiers, ed. A.J. Smola, P.L. Bartlett, B. Schölkopf, and D. Schuurmans, The MIT Press, Cambridge, MA, 2000.

[28] R. Rifkin, G. Yeo, and T. Poggio, "Regularized least-squares classification," Advances in Learning Theory: Methods, Models and Applications, ed. J.A.K. Suykens, G. Horvath, S. Basu, C. Micchelli, and J. Vandewalle, NATO Science Series III: Computer \& Systems Sciences, vol.190, pp.131-154, IOS Press, Amsterdam, The Netherlands, 2003.

[29] M. Schmidt, minFunc, 2005. http://people.cs.ubc.ca/ ${ }^{\text {schmidtm/ }}$ Software/minFunc.html

[30] B. Schölkopf and A.J. Smola, Learning with Kernels, MIT Press, Cambridge, MA, 2002. 
[31] J.A.K. Suykens, T.V. Gestel, J.D. Brabanter, B.D. Moor, and J. Vandewalle, Least Squares Support Vector Machines, World Scientific Pub. Co., Singapore, 2002.

[32] Y. Tang and H.H. Zhang, "Multiclass proximal support vector machines," J. Computational and Graphical Statistics, vol.15, no.2, pp.339-355, 2006.

[33] C.H. Teo, Q. Le, A. Smola, and S.V.N. Vishwanathan, "A scalable modular convex solver for regularized risk minimization," ACM SIGKDD International Conference on Knowledge Discovery and Data Mining (KDD2007), pp.727-736, 2007.

[34] I. Tsang, J. Kwok, and P.M. Cheung, "Core vector machines: Fast SVM training on very large data sets," J. Machine Learning Research, vol.6, pp.363-392, 2005.

[35] V.N. Vapnik, Statistical Learning Theory, Wiley, New York, 1998.

[36] T.F. Wu, C.J. Lin, and R.C. Weng, "Probability estimates for multiclass classification by pairwise coupling," J. Machine Learning Research, vol.5, pp.975-1005, 2004.

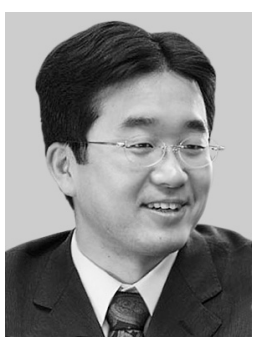

Masashi Sugiyama received the B.E., M.E., and Ph.D. degrees from Department of Computer Science, Tokyo Institute of Technology, Tokyo, Japan, in 1997, 1999, and 2001. In 2001, he was appointed as a Research Associate in the same institute, and from 2003, he is an Associate Professor. His research interests include theory and application of machine learning and signal/image processing. 